\title{
Analysis of CALET Data for Anisotropy in Electron+Positron Cosmic Rays
}

\author{
Holger Motz* \\ Global Center for Science and Engineering, Faculty of Science and Engineering, \\ Waseda University, 3-4-1 Okubo, Shinjuku, Tokyo 169-8555, Japan \\ E-mail: motzeaoni.waseda.jp
}

\section{Yoichi Asaoka}

Waseda Research Institute for Science and Engineering, Faculty of Science and Engineering,

Waseda University, 3-4-1 Okubo, Shinjuku, Tokyo 169-8555, Japan

E-mail: yoichi.asaoka@aoni.waseda.jp

\section{for the CALET collaboration ${ }^{\dagger}$}

The ISS-based Calorimetric Electron Telescope (CALET) is directly measuring the energy spectrum and direction distribution of electron+positron cosmic-rays up to $20 \mathrm{TeV}$. A main goal of CALET is to identify a signature of a nearby supernova remnant in electron+positron cosmic-rays, such as a spectral feature in the $\mathrm{TeV}$ region and/or a detectable anisotropy. The electron+positron events measured by CALET have been analyzed for a possible dipole anisotropy, as well as higher multipole moments. The methods for deriving limits on the anisotropy from the reconstructed events, as well as the procedures to compensate for the non-uniform exposure to the sky and inhomogeneous acceptance of the detector are explained. Preliminary results for the measured anisotropy and upper limits depending on threshold energy are presented, together with sensitivity estimations and a comparison to the expected anisotropy caused by the Vela supernova remnant

36th International Cosmic Ray Conference -ICRC2019-

July 24th - August 1st, 2019

Madison, WI, U.S.A.

\footnotetext{
${ }^{*}$ Speaker.

${ }^{\dagger}$ for collaboration list see PoS(ICRC2019)1177
} 


\section{Introduction}

During recent years, the precision of measurements of the electron + positron cosmic-ray spectrum has increased significantly, with space based direct measurements by CALET $[1,2]$ and DAMPE [3] reaching into the $\mathrm{TeV}$ energy region, which is expected to be dominated by cosmic rays emitted by very nearby supernova remnants (SNR), since radiative energy loss limits the propagation distance of electron cosmic rays reaching Earth at $\mathrm{TeV}$ energy to less than one kiloparsec $(\mathrm{kpc})$ [4]. Detecting a signature of cosmic-ray acceleration in the nearby SNR is one of the main physics goals of CALET [5], and searching for the dipole anisotropy expected from a dominating single source in the measured flux in addition to spectral information is an important tool to achieve this goal. The preliminary analysis presented herein aims mainly at establishing the methods and to ensure the correct understanding of the detector response to allow testing for actual signatures using further increased statistics of flight data in combination with the also continuously extending spectral measurement [6].

\section{Method of Analysis}

The analysis for a dipole or higher multipole anisotropy in CALET electron+positron data is based on the methods used by the Fermi-LAT collaboration for analysis of their data as described in Ref. [7]. The HEALPix [8] anafast routines are used to perform a spherical harmonic decomposition of the event distribution on the sky in galactic coordinates, calculating the multipole coefficients $C_{l}$ for monopole $\left(C_{0}\right)$, dipole $\left(C_{1}\right)$, quadrupole $\left(C_{2}\right)$ and octupole $\left(C_{3}\right)$. From these coefficients, the dipole magnitude $\delta$ is calculated as

$$
\delta=3 \sqrt{\frac{C_{1}}{C_{0}}}
$$

For an event distribution with negligible higher multipole moments this corresponds to the ratio of the largest flux difference to twice the average flux,

$$
\delta \approx \frac{\Phi_{\max }-\Phi_{\min }}{\Phi_{\max }+\Phi_{\min }}
$$

While the right side of Eq. 2.2 has a maximum of one, $\delta$ from Eq. 2.1 can be larger for distributions more sharply peaked in one direction than a dipole, and is three for a signal from a single direction. As a gradient in the cosmic-ray density caused by propagation from a nearby source would result in a dipole anisotropy [9], the approximation is valid for the maximal expected concentration of events in one direction, but values of $\delta>1$ appear in distributions with small event numbers due to statistical fluctuation (the combined monopole and dipole components are negative in opposite direction to the event concentration, which is compensated by contribution of higher multipoles).

The analysis is performed on samples of events above a threshold energy for which five values equidistant in $\log$ scale between $125 \mathrm{GeV}$ and $2 \mathrm{TeV}$ are chosen. Owing to the steep spectrum, each sample is dominated by events close to the threshold. 
From the measured value of $\delta_{\text {meas }}$, the $95 \%$ CL limit is calculated as the value of $\delta$ for which the probability of the measured and smaller anisotropy is five percent, i.e. by solving the integral

$$
\frac{3 \sqrt{6}}{\sqrt{\pi} \delta^{3}} \int_{0}^{\delta_{\text {meas }}} \hat{\delta}^{2} e^{-\left(\frac{3 \hat{\delta}^{2}}{2 \delta^{2}}\right)} d \hat{\delta}=0.05
$$

numerically [7].

\section{Data Sample and Exposure Correction}

The data taken into account contains all electron candidate events above an reconstructed energy of $125 \mathrm{GeV}$ from the start of scientific observation on 2015/10/13 until 2018/10/31, 1115 days in total, using the boosted decision tree electron separation method. To compensate for the non-uniform exposure of the sky to CALET and the direction-dependent reconstruction efficiency, the events are weighted relative to the average exposure and reconstruction efficiency. The effective area for any given direction in the detector coordinate system is calculated as a HEALPix map, in which all the event-selection efficiencies are included. Along the ISS orbit, the effective area for any given direction is projected to the sky in the galactic coordinate system weighted by the live time. As a result, an exposure map is obtained for any given period of observation, depending also on energy and the event classification, which is described in the supplemental material of Ref. [2]. The exposure maps use the same binning as the HEALPix maps containing the events (HEALPix resolution parameter $N_{\text {side }}=64, \approx 50 \mathrm{k}$ pixels [8]) and are averaged over the observation time for the data sample. Each event receives a weight $w_{i}$ given by

$$
w_{i}=\frac{1}{A_{i}}\left(\frac{\sum_{\text {events pixels }} A(E, \text { pixel })}{\left.N_{\text {events }} N_{\text {pixels }}\right)}\right)
$$

where $A_{i}$ is the individual exposure for the direction and energy of the event.

For each sample of events above the respective threshold energy, an average exposure map is composed by combining the exposure maps for the events in the sample. These average exposure maps for which examples are given in Figure 1 are used to include the influence of uneven exposure and exposure correction in the Monte Carlo simulation for estimation of expected anisotropy from statistical fluctuation.

\section{Comparison Models}

To put the results of the anisotropy analysis into context, they are compared to predictions by a model interpreting available cosmic-ray spectral data as explained in the following. The statistical fluctuation of event directions result in the occurrence of multipole moments in the measurement also for a purely isotropic spectrum, based on the number of events in the sample. To estimate these fluctuations, the electron+positron flux is modeled and used to create statistical samples. As a modification to the model, the high energy part of the electron+positron spectrum is assumed to originate purely from the Vela SNR, to estimate the potential anisotropic component of the flux in this energy region, into which also the cosmic-ray propagation conditions enter. 

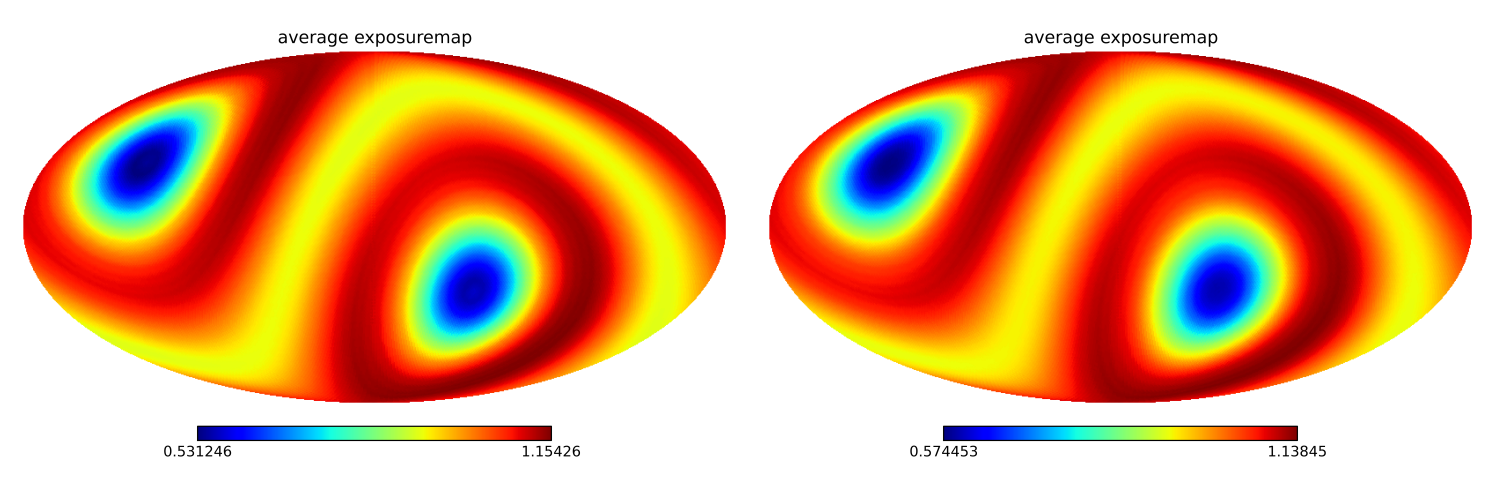

Figure 1: Average and normalized exposure maps for $125 \mathrm{GeV}$ (left) and $1 \mathrm{TeV}$ (right) threshold energy.

The chosen propagation conditions (also used in [10]) comprise a gradual change in the slope of the diffusion coefficient according to

$$
D(r)=D_{0}\left(\frac{R}{R_{0}}\right)^{\delta_{l}}\left(1+\left(\frac{R}{R_{b}}\right)^{\frac{\delta_{l}-\delta_{h}}{s}}\right)^{-s}
$$

with $\delta_{l}=0.6, \delta_{h}=0.33, R_{0}=4 \mathrm{GV}, R_{b}=300 \mathrm{GV}, D_{0}=1.3 \cdot 10^{28} \mathrm{~cm}^{2} / \mathrm{s}$, and a softness parameter $s=0.2$. In calculation of nuclei spectra with DRAGON [11], assuming a common injection index $\gamma_{i}=-2.32$ for all nucleons, this model reproduces the AMS-02 B/C ratio [12] and proton spectrum [13] measurements, predicting a hardening in the proton spectrum, albeit too weak to be the sole explanation for the magnitude of index change as recently measured by CALET $[14,15]$ (an adaption of the model is currently being investigated).

Due to their large energy loss in propagation, the electron and positron spectra depend on the exact distribution of individual point sources. Modeling the precise measurements of AMS02 [16] and CALET [2] requires to fine-tune the parameters, which can be achieved better with a partly analytic parametrization. The parametrization is based on a power law with a soft spectral break (index $\gamma_{e}$, break position $E_{b}$, index change $\Delta \gamma$, softness $s$ ) at low energy and an high-energy exponential cut-off at $E_{c u t_{d}}$ representing directly the propagated spectrum of primary electrons from SNR. Secondary positron and electron fluxes are taken from the DRAGON results for nuclei, with a common scaling factor $\left(C_{s} / C_{n o r m}\right)$ as free parameter. The positron excess is attributed to multiple nearby pulsars (selected by their potential contribution to the total flux being more than 5\%) with position, age and total rotation energy taken from the ATNF catalog [17], while injection efficiency $\eta$, spectral index $\gamma_{e x}$ and cut-off energy $E_{c u t_{e x}}$ are free parameters common for all pulsars. It is assumed that the high energy electrons and positrons are confined in the PWN initially and instantly released with the dissolving of the PWN after $T_{r}=60 \mathrm{kyr}[18]$. The injection spectra are propagated using the analytic solution of the propagation equation for a point source as explained e.g. in Ref. [19]. This parameter fit reproduces the lepton spectra very well as demonstrated in Figure 2. (A more detailed explanation of the model and parameter fitting is given in Ref. [20].) 


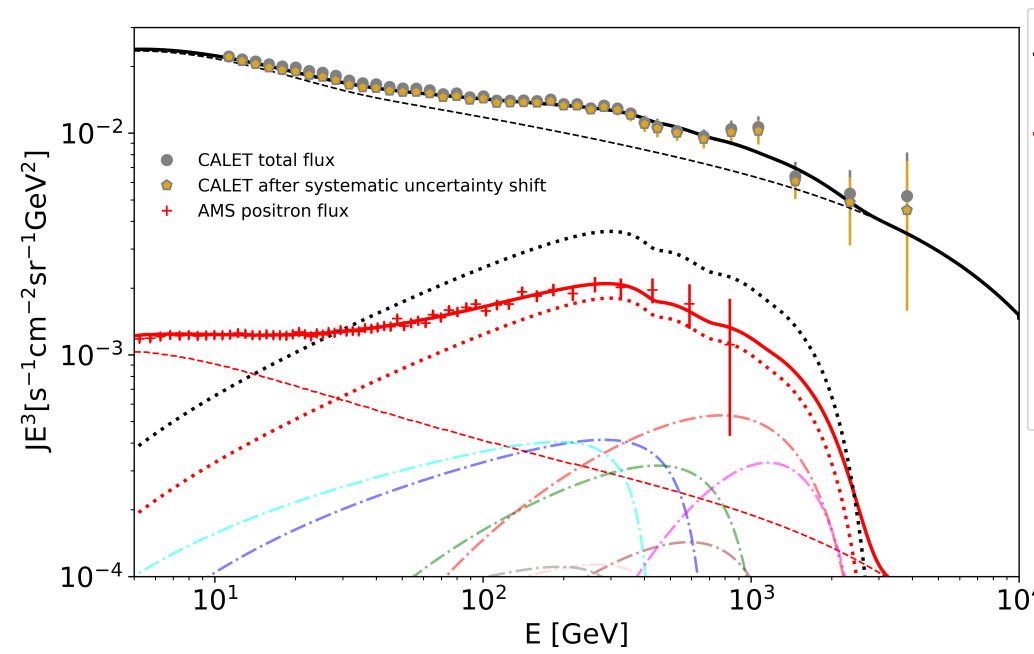

Parametrization Fit $\left(\chi^{2} /\right.$ ndof $\left.=26.2 / 77.0\right)$

- total flux, $\chi^{2}: 14.3$ (CALET data)

---- background from secondaries + distant SNR

$\cdots$ total flux from all pulsars

- positron flux, $x^{2}: 12.0$ (AMS-02 data)

---- background from secondaries

.. positron/electron flux from all pulsars

Individual Pulsars:

J0633+1746 [Geminga]

j0659+1414 [Monogem]

- J0954-5430

J1057-5226

- J1732-3131

J1741-2054

J1745-3040

Parameter Values:

$\mathrm{C}_{\mathrm{e}}=0.0800 \mathrm{~cm}^{-2} \mathrm{~s}^{-1} \mathrm{sr}^{-1} \mathrm{GeV}^{-1}$

$\Delta \mathrm{Y}=0.25 ; \mathrm{E}_{\mathrm{B}}=33.39 \mathrm{GeV} ; \mathrm{s}=0.05$

$C_{s} / C_{\text {norm }}=1.33$
$\eta=0.003 ; Y_{e x}$

$T_{r}=60.00 \mathrm{kyr}=1.81 ; \mathrm{E}_{\text {cut }_{\text {ex }}}=1371 \mathrm{GeV}$

$\mathrm{E}[\mathrm{GeV}]$

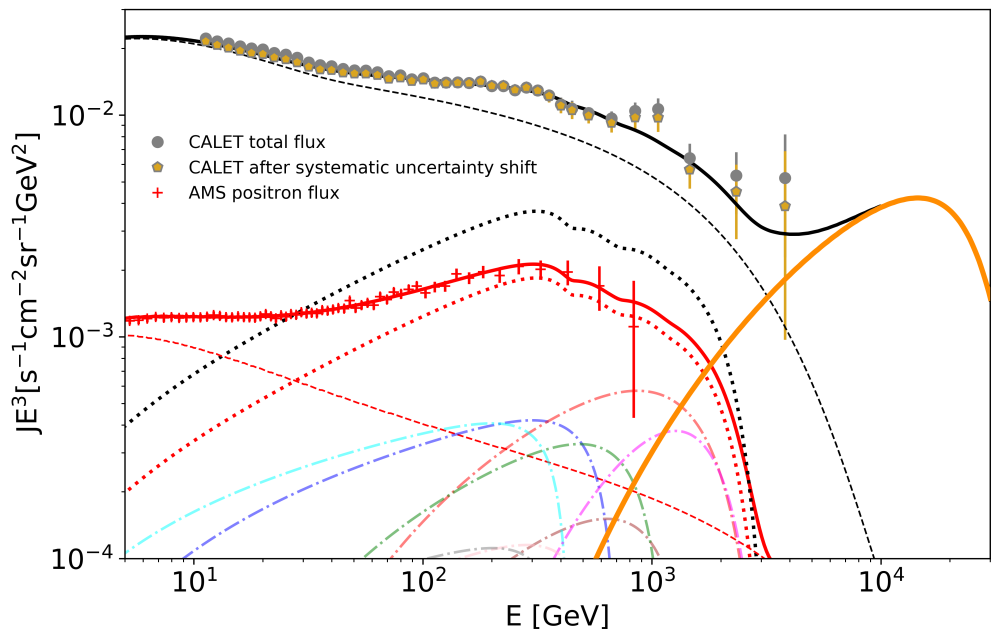

Parametrization Fit $\left(\chi^{2} /\right.$ ndof $\left.=28.4 / 76.0\right)$

total flux, $x^{2}: 16.2$ (CALET data)

background from secondaries + distant SNR total flux from all pulsars

positron flux, $\chi^{2}: 12.2$ (AMS-02 data)

background from secondaries

positron/electron flux from all pulsars

electron flux from local SNR (Vela)

J0633+1746 [Geminga]

J0659+1414 [Monogem]

J0954-5430

J1003-4747

J1057-5226

J1732-3131

- J1745-3040

Parameter Values:

$\mathrm{C}_{\mathrm{e}}=0.0689 \mathrm{~cm}^{-2} \mathrm{~s}^{-1} \mathrm{sr}^{-1} \mathrm{GeV}^{-1}$

$\mathrm{V}_{\mathrm{e}}=3.17 ; \mathrm{E}_{\mathrm{cut}_{\mathrm{d}}}=2000 \mathrm{GeV}$

$\Delta \gamma=0.26 ; E_{B}=38.64 \mathrm{GeV} ; s=0.05$

$\mathrm{C}_{\mathrm{s}} / \mathrm{C}_{\text {norm }}=1.31$

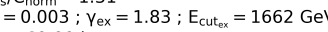

$\mathrm{T}_{\mathrm{r}}=60.00 \mathrm{kyr}$

Figure 2: Models of electron+positron flux by parameter fitting to CALET [2] and AMS-02 [16] data. See legends for explanation of markers and lines, the values for the parameters introduced in the text are given in the box to the right of the graphs. The model shown in the upper panel is used as reference for the flux of an isotropic distribution, for the model in the lower panel the anisotropy of the Vela SNR is calculated and compared to limits.

The flux predicted by this model is used together with the averaged exposure maps to create samples of CALET data for the case of an isotropic cosmic-ray flux. The flat expectation map is multiplied with the exposure map before randomly assigning the event number in each pixel according to the Poisson distribution for the expected number of events. For each threshold energy, 20000 samples are created and analyzed in the same way as the flight data, yielding the expectation ranges shown in the figures of the result section.

The flux in the SNR region could be dominated by a single source, with the Vela SNR being the main candidate, due to its distance of $0.3 \mathrm{kpc}$ and age of $11000 \mathrm{yr}$ [4]. The above model is modified 
to show the dipole anisotropy expected in this case. The expected anisotropy from the Vela SNR as presented for comparison to limit results in Figure 3 is calculated using DRAGON, which features a non-equidistant spatial grid, allowing for a fine spatial binning near the observation point and sources required for calculation of anisotropy. The directional derivative of the cosmic ray density $F(x, y, z)$, is expressed as a difference between grid points with positions $\vec{r}_{1}$ and $\vec{r}_{2}$ on opposite sides of the Solar System's position $\vec{r}_{s u n}$. The flux $\Phi_{d i r}$ from direction of the grid point at position $\vec{r}_{1}$ is calculated as

$$
\Phi_{d i r}=\Phi_{\text {avg }}\left(1+\frac{3 D(R)}{c} \frac{F\left(\vec{r}_{1}\right)-F\left(\vec{r}_{2}\right)}{\left|\vec{r}_{1}-\vec{r}_{2}\right| F\left(\vec{r}_{\text {sun }}\right)}\right) .
$$

The injection spectrum of Vela SNR is given by power law index $\gamma_{i}=-2.32$ and cut-off energy $E_{c}=100 \mathrm{TeV}$, assuming constant emission over 5000 years. The total energy of emitted cosmic-ray electrons with $\mathrm{E}>1 \mathrm{GeV}$ is normalized to $2.5 \cdot 10^{47} \mathrm{erg}$, to be compatible with CALET data.

\section{Results}

Figure 3 shows the measured dipole anisotropy and resulting 95\% CL limits together with the expectation ranges. The results fall within the 2- $\sigma$ boundary of the expectation for statistical fluctuations of an isotropic cosmic-ray flux.

Owing to the larger effective area of Fermi-LAT, the limits from Ref. [7] are more stringent in the energy range for which they are applicable. The Fermi-LAT 7-year anisotropy limits published in Ref. [21] are for independent energy bins, not threshold energies, and not directly comparable, though certainly much more stringent below $1 \mathrm{TeV}$ than the obtained CALET limits. However, since the anisotropy found for each bin is dominated by the larger number events in the lower energy part of the bin, the limit set by the highest energy bin is not directly applicable for the TeV-region, making the presented limits from CALET data the first explicit constraint of electron+positron flux dipole anisotropy in this energy range.

The predicted anisotropy of the above explained model for the Vela SNR is not ruled out by the Fermi-LAT limit as shown in Figure 4, demonstrating that a yet undetected significant dipole anisotropy could exist in the $\mathrm{TeV}$ energy region, making further search for anisotropy in this energy range worthwhile.

In addition to the dipole amplitude, the quadrupole and octupole moments were analyzed, with the former being a useful tool to investigate the influence of the exposure correction. While there is only limited influence on the obtained and expected results for the dipole amplitude, the non-uniformity of the exposure is quadrupole-shaped and thus will imprint a quadrupole structure on an isotropic event distribution. This structure is detected at low energy as expected from the simulation if not applying the exposure correction in the analysis, while the quadrupole moment is reduced to the level expected from random fluctuations when applying the exposure correction, proving the reliability of the correction. The octupole moment is not influenced by the exposure correction and measured values fall well within the expectation range.

\section{Summary}

The analysis of CALET data from about three years of flight data yields results on the dipole magnitude agreeing to what is expected from statistical fluctuations of an isotropic electron+positron 


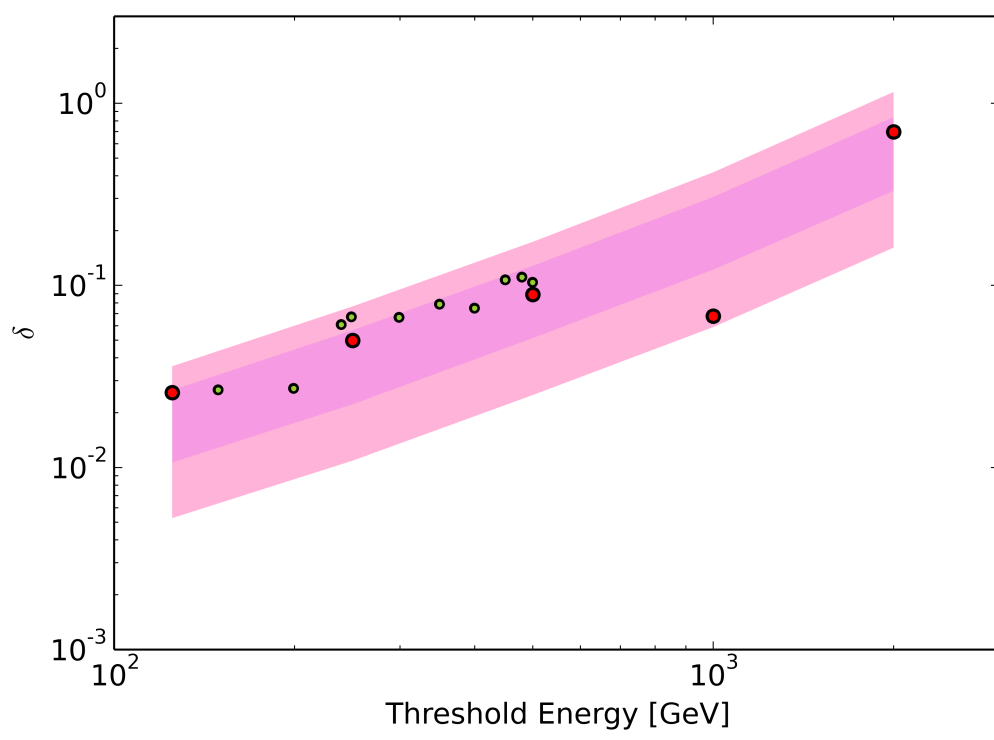

- Measured anisotropy of 1115-day CALET data $1 \sigma$ region of expected measured anisotropy for 1115-day data $2 \sigma$ region of expected measured anisotropy for 1115-day data

- $\quad 95 \%$ CL Fermi-LAT

- limits 2010

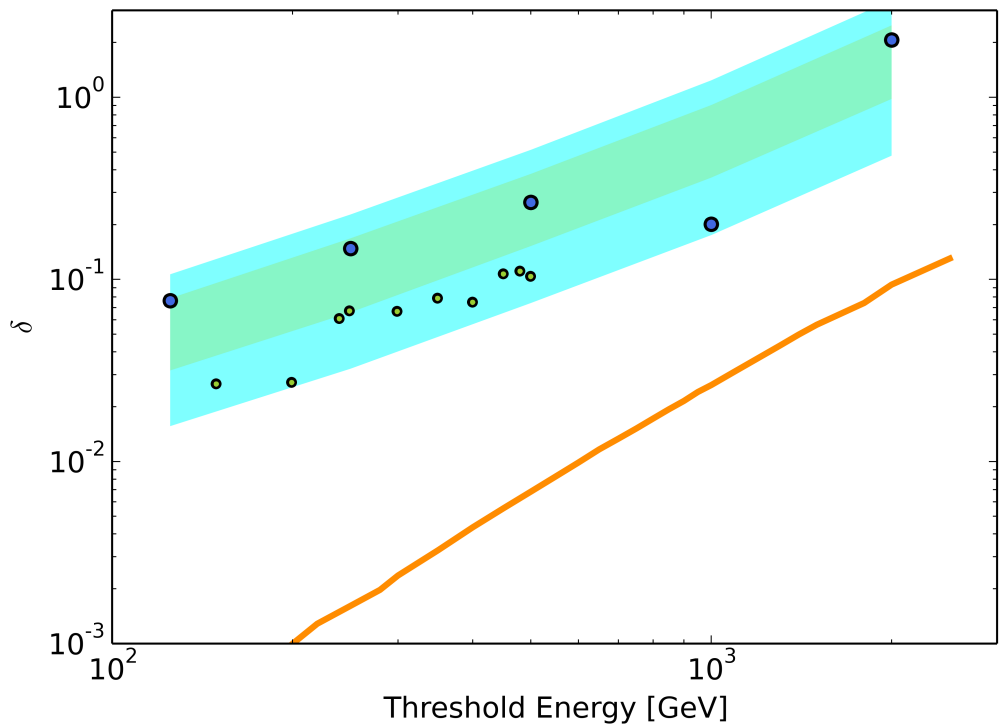

Measured 1115-day 95\%CL CALET limit $1 \sigma$ region of expected 1115-day 95\%CL limit $2 \sigma$ region of expected 1115-day 95\%CL limit Predicted anisotropy for Vela SNR model

95\% CL Fermi-LAT

- limits 2010

Figure 3: Measured and expected dipole anisotropy (top) and 95\% CL limit (bottom) as a function of threshold energy, compared to limits by Fermi-LAT [7].

cosmic-ray flux. These preliminary results set limits on the dipole anisotropy in the $\mathrm{TeV}$ region, not yet explicitly covered by other experiments.

A significant anisotropy could exist at $\mathrm{TeV}$ energy from electrons accelerated by the Vela SNR for standard emission and propagation conditions meeting the recently tightening experimental constraints. This presents a target for further measurements of CALET and future analyses with increased flight data statistics and refined methods potentially combining spectral and anisotropy information to enhance sensitivity. 


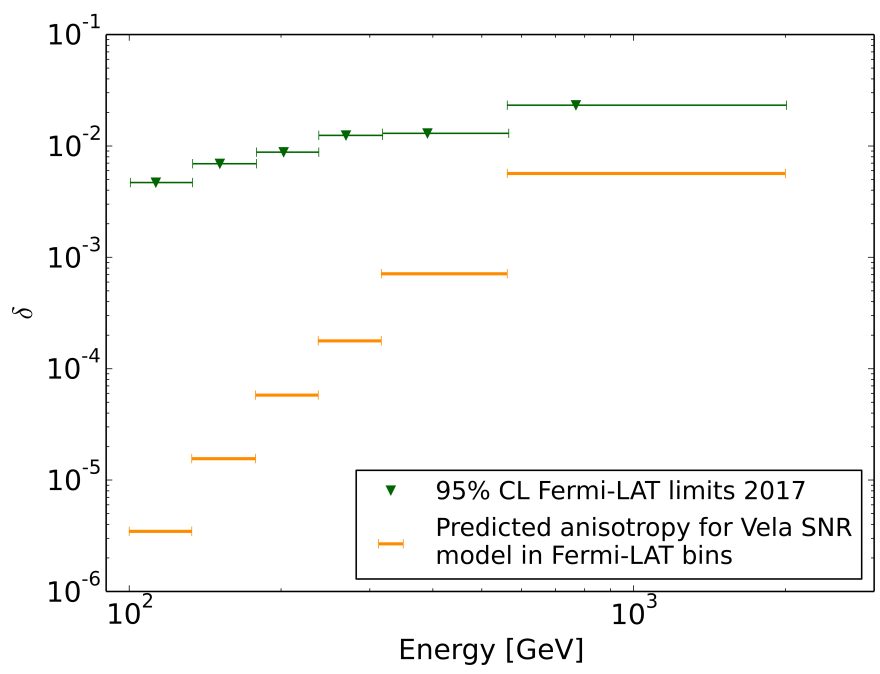

Figure 4: Predicted dipole anisotropy for the Vela SNR model shown in the lower panel of Figure 2, compared to Fermi-LAT limits from Ref. [21].

\section{References}

[1] O. Adriani, et al., Phys. Rev. Lett. 119, 181101 (2017).

[2] O. Adriani, et al., Phys. Rev. Lett. 120, 261102 (2018).

[3] G. Ambrosi, et al., Nature 552, 63 (2017).

[4] T. Kobayashi, Y. Komori, K. Yoshida, J. Nishimura, Astrophys. J. 601, 340 (2004).

[5] Y. Asaoka, et al., PoS ICRC2019 (2019). In press.

[6] S. Torii, et al., PoS ICRC2019 (2019). In press.

[7] M. Ackermann, et al., Phys.Rev. D82, 092003 (2010).

[8] K. M. Górski, et al., Astrophys. J. 622, 759 (2005).

[9] K. Yoshida, International Cosmic Ray Conference 6, 367 (2011).

[10] H. Motz, Y. Asaoka, S. Torii, S. Bhattacharyya, PoS ICRC2017, 265 (2018).

[11] D. Gaggero, L. Maccione, G. Di Bernardo, C. Evoli, D. Grasso, Phys.Rev.Lett. 111, 021102 (2013).

[12] M. Aguilar, et al., Phys. Rev. Lett. 117, 231102 (2016).

[13] M. Aguilar, et al., Phys. Rev. Lett. 114, 171103 (2015).

[14] O. Adriani, et al., Phys. Rev. Lett. 122, 181102 (2019).

[15] P. Marrocchesi, et al., PoS ICRC2019 (2019). In press.

[16] M. Aguilar, et al., Phys. Rev. Lett. 122, 041102 (2019).

[17] R. N. Manchester, G. B. Hobbs, A. Teoh, M. Hobbs, Astron.J. 129, 1993 (2005).

[18] D. Malyshev, I. Cholis, J. Gelfand, Phys.Rev. D80, 063005 (2009).

[19] J. Feng, H.-H. Zhang, The European Physical Journal C 76, 229 (2016).

[20] H. Motz, Y. Asaoka, S. Bhattacharyya, PoS ICRC2019 (2019). In press.

[21] S. Abdollahi, et al., Phys. Rev. Lett. 118, 091103 (2017). 